\title{
Adenopatías mediastínicas en la evolución de un cáncer de próstata metastásico
}

\author{
Roca Edreira A, Aguilera Tubet C, Villanueva Peña A, Ballestero Diego R, Zubillaga Guerrero S.
}

Servicio de Urología. Hospital Universitario Marqués de Valdecilla. Santander. Cantabria.

Actas Urol Esp. 2007;31(6):693-695

\section{RESUMEN}

\section{ADENOPATIAS MEDIASTINICAS EN LA EVOLUCION DE UN CANCER DE PROSTATA} METASTASICO

El cáncer de próstata es uno de los tumores más frecuentes. Un número importante de pacientes presentarán adenopatías regionales y metástasis óseas en el curso de la enfermedad. Sin embargo, las adenopatías mediastínicas y las metástasis cutáneas son infrecuentes y significan enfermedad avanzada. Presentamos el caso de un paciente diagnosticado de cáncer de próstata que desarrolla adenopatías mediastínicas, nódulos pulmonares y metástasis cutáneas, 8 años después del diagnóstico.

Palabras clave: Cáncer de próstata. Metástasis óseas. Adenopatías.

\section{ABSTRACT}

MEDIASTINAL LYMPH NODES DURING THE COURSE OF A METASTATIC PROSTATE CANCER Prostate carcinoma is one of the most frecuent cancers in men. Significant numbers of patients have regional lymph node and bone metastases during the course of the disease. Mediastinal lymphadenopathy and cutaneous metastases are uncommon and signify well-advanced disease. We report the case of a patient with prostate cancer who develops mediastinal lymphadenopathy, pulmonary nodules and cutaneous metastases 8 years after the diagnosis.

Keywords: Prostate cancer. Bone metastases. Lymphadenopathy.

$\mathrm{E}^{\prime}$ cáncer de próstata tiene una prevalencia muy alta, siendo el cuarto en orden de frecuencia a nivel mundial. En la evolución de la enfermedad, la mayor parte de los pacientes desarrollan adenopatías regionales, pélvicas, y metástasis óseas, y en casos avanzados pueden llegar a presentar metástasis pulmonares. Sin embargo, es excepcional el desarrollo de adenopatías mediastínicas y cutáneas, como en el caso que comunicamos.

\section{MATERIAL Y METODOS}

El caso que nos ocupa es el de un paciente remitido a nuestra consulta a raíz de un episodio de infección urinaria, con un PSA de 380. Previamente al episodio refiere un síndrome prostático discreto y la próstata a la exploración, era grande y aumentada de consistencia en ambos lóbulos. Como antecedentes personales sólo destaca hipertensión arterial e hiperuricemia. Ante los datos clínicos se realiza una biopsia de próstata guiada por ecografia transrectal y una gammagrafía ósea.

La biopsia confirma un adenocarcinoma prostático acinar grado III de MDA (4+4 de Gleason) en todos los cilindros recibidos de los dos lóbulos. La gammagrafía ósea informa de un foco hipercaptador sugestivo de metástasis en isquion derecho.

Ante el diagnostico de adenocarcinoma de próstata metastásico, se comienza tratamiento con flutamida y triptorelina trimestral. El PSA disminuye hasta 0,17 a los 8 meses, pero es necesario 
cambiar la flutamida por ciproterona debido a un aumento de las enzimas hepáticas, con lo que estas vuelven a normalizarse. $\mathrm{El}$ paciente permanece con un PSA estable en torno a 0,10 durante dos años, periodo tras el cual comienza a elevarse pese a la suspensión del antiandrógeno, y posteriormente de la triptorelina, comenzando tratamiento con estramustina.

Tras cuatro años desde el diagnóstico y con un PSA de 387, la fosfatasa alcalina ósea comienza a elevarse, y en la radiografia simple de pelvis se ven metástasis osteoblásticas, comenzando el paciente a tener dolores óseos diseminados y a estar ictérico. En este momento se inicia tratamiento con zoledronato cálcico y vitamina $\mathrm{D}$, suspendiéndose la estramustina. Durante los siguientes 3 años, el PSA va subiendo hasta situarse en 7385. El tratamiento fue variando con antiandrógeno, análogo de LHRH, estramustina y por último ketoconazol. $\mathrm{El}$ paciente recibió varios ciclos de tratamiento con zoledronato, cuando refería dolor óseo, que cedía con antiinflamatorios no esteroideos.

A los 8 años del diagnostico, el paciente presenta edema sin fovea hasta las caderas y en la ingle derecha, presenta unos nódulos duros en la piel, muy sugestivos de metástasis cutáneas de adenocarcinoma de próstata. Al mes, se realiza un TAC de tórax por sospecha de tumor de Pancoast. En él se describen adenopatías a nivel mediastínico y en localización hiliar derecha, asî como lesiones nodulares múltiples a nivel del parénquima pulmonar sugestivas de metástasis y derrame pleural derecho (Figs. 1, 2 y 3).

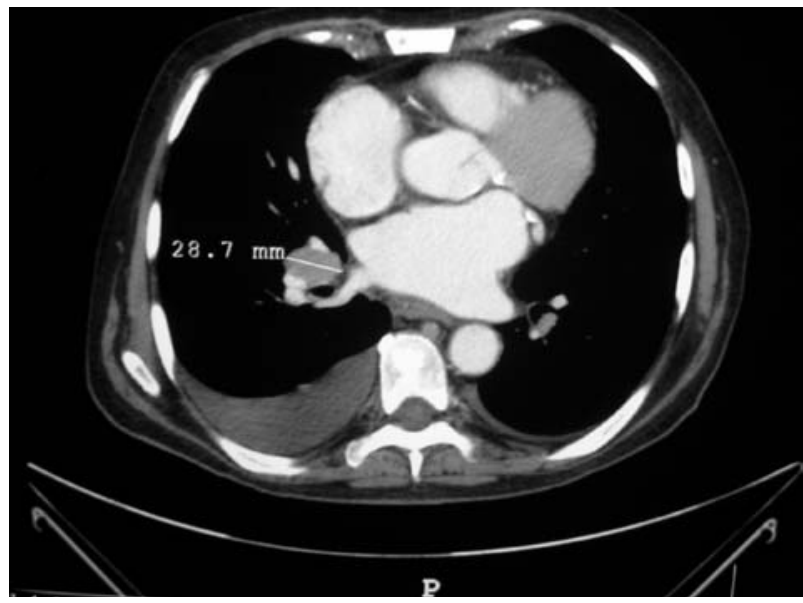

FIGURA 1. Adenopatia hiliar derecha.

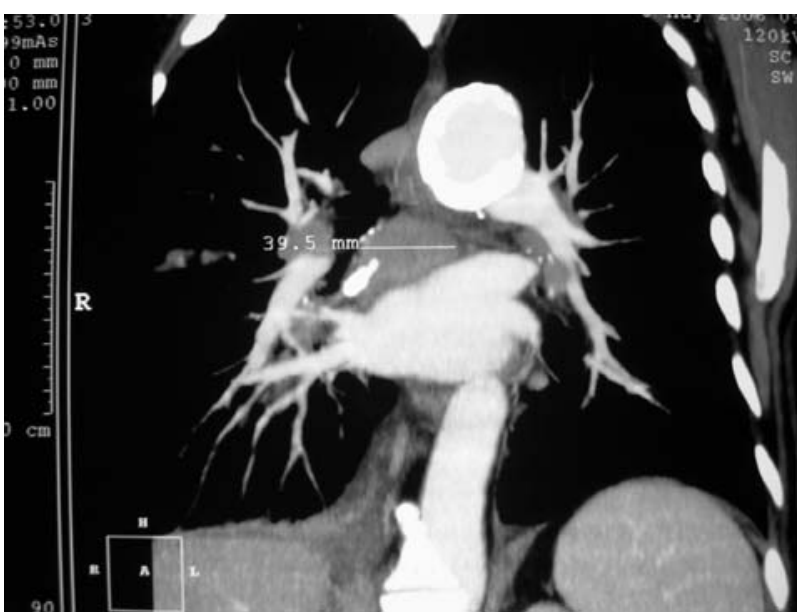

FIGURA 2. Adenopatias mediastinicas e hiliar derecha.

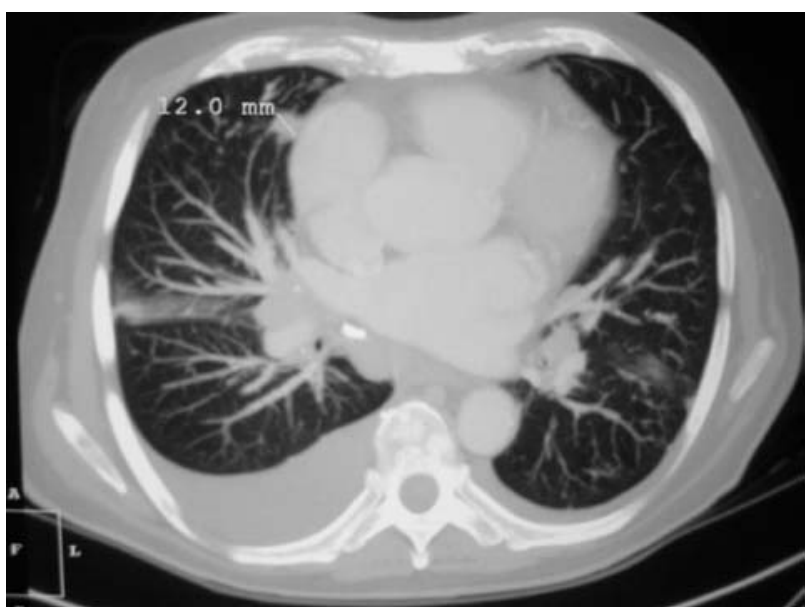

FIGURA 3. Nódulos pulmonares.

A los tres meses, el paciente acude al servicio de urgencias con dolor lumbar que se irradia por la extremidad inferior derecha de dificil control con la analgesia pautada, junto con disnea. Al ingreso, el paciente estaba anémico, precisando la transfusión de dos concentrados de hematíes, y tenia un PSA de 26.840. A las horas desarrolla un cuadro de dificultad respiratoria falleciendo.

\section{DISCUSION}

El cáncer de próstata es la cuarta neoplasia en orden de frecuencia a nivel mundial. Con el desarrollo del PSA como método de screenning, aumenta la incidencia de enfermedad locorregional en el momento del diagnostico, mientras que disminuye la de enfermedad metastásica ${ }^{1}$.

El caso que nos ocupa se trata de enfermedad metastásica ya desde el momento del diagnóstico, con una metástasis ósea. Tras dos años de bloqueo 
hormonal máximo, el tumor se vuelve hormonorresistente, y dos años después comienza a elevarse la fosfatasa alcalina ósea, presentando entonces metástasis óseas diseminadas.

Hasta este momento, la evolución que sigue nuestro paciente es la normal en la historia natural del cáncer de próstata metastásico. Sin embargo, el desarrollo de adenopatías no regionales, mediastínicas, es extremadamente infrecuente en el curso natural de esta enfermedad. De este modo, la mayor parte de las adenopatías mediastínicas se observan en pacientes en quienes el tumor primario se encuentra en el tórax. De hecho, Lindell et al. comunican 7 de 1.435 pacientes $(0,49 \%)$ que desarrollan adenopatías mediastínicas durante el curso de la enfermedad ${ }^{2}$. Boyer et al. comunican un caso similar al nuestro, en que el paciente presenta adenopatías mediastínicas que producen compresión de estructuras vecinas ${ }^{3}$.

En cuanto a la significación diagnóstica del desarrollo de adenopatías mediastínicas, estas han de ser consideradas como metástasis a distancia, según la clasificación TNM de 1997. Furuya et al. estudiaron la significación pronóstica de estas en 205 pacientes con cáncer de próstata metastásico. Concluyen que la terapia endocrina es efectiva incluso en pacientes con adenopatías no regionales ${ }^{4}$.

Adicionalmente, el paciente desarrolla nódulos pulmonares y cutáneos múltiples sugestivos de metástasis por adenocarcinoma de próstata. El desarrollo de metástasis pulmonares en los estadíos avanzados del cáncer de próstata no es un hecho infrecuente, pero si lo es el desarrollo de metástasis cutáneas ${ }^{5,6}$.

Menos de 5\% de tumores de órganos internos metastatizan en la piel, y el cáncer de próstata representa un porcentaje muy pequeño de estos.
Estas lesiones suelen aparecer tarde en la evolución de la enfermedad, y son signo de mal pronóstico. Las lesiones son sólidas, en ocasiones ulceradas y se suelen localizar en el abdomen bajo y los genitales ${ }^{7}$.

En el caso que nos ocupa, no se realizó ningún estudio invasivo para confirmar el origen anatomopatológico de las lesiones mediastínicas ni cutáneas, dado que la sospecha era muy alta por la evolución de la enfermedad con metástasis óseas desde el diagnóstico, un PSA de 26.840 y que la imagen tomográfica del tórax era muy sugestiva de metástasis no encontrándose otro tumor primario en el tórax, en un paciente con mala situación clínica.

\section{REFERENCIAS}

1. Newcomer LM, Stanford JL, Blumenstein BA, Brawer MK. Temporal trends in rates of prostate cancer: Declining incidence of advanced stage disease, 1974 to 1994 . J Urol 1997;158(4): 1427-1430.

2. Lindell MM, Doubleday LC, von Eschenbach AC, Libshitz HI. Mediastinal metastases from prostatic carcinoma. J Urol 1982;128(2):331-224.

3. Boyer L, Fraysse P, Taillandier J, Michel JL. Mediastinal adenopathy and cancer of the prostate. Rev Mal Respir 1991;8(1):115117.

4. Furuya Y, Akakura K, Akimoto S, Ito H. Prognosis of patients with prostate carcinoma presenting as nonregional lymph node metastases. Urol Int 1998;61(1):17-21

5. Mestitz H, Pierce RJ, Holmes PW. Intrathoracic manifestations of disseminated prostatic adenocarcinoma. Respir Med. 1989; 83(2): 161-166.

6. Landow RK, Rhodes DW, Bauer M. Cutaneous metastases. Report of two cases of prostatic cancer. Cutis. 1980;26(4):399-401.

7. Azana JM, de Misa RF, Gómez MI, del Hoyo JF, Ledo A. Cutaneous metastases from prostatic cancer. J Dermatol. 1993;20 (12):786-788.

Correspondencia autor: Dr. A. Roca Edreira

Servicio de Urología. Hospital Universitario Marqués de Valdecilla Avda. de Valdecilla, s/n - 39008 Santander

Tel.: 942202520

E-mail autor: anroed@ono.com

Información artículo: Nota clínica

Trabajo recibido: abril 2007

Trabajo aceptado: mayo 2007

\section{COMENTARIO EDITORIAL}

Quisiera agradecer a los autores la publicación de este caso que refleja la evolución de un adenocarcinoma de próstata hormonorresistente. De todas formas, dado que el caso se presentaba con derrame pleural, y estando de acuerdo en la actitud de ser lo menos agresivo posible, existe la posibilidad de diagnosticar de diagnosticar la diseminación pulmonar de un cáncer de próstata mediante el análisis del líquido pleural ${ }^{1}$. Hecha esta salvedad de nuevo mi enhorabuena por la exposición.

\section{REFERENCIAS}

1. Sánchez Merino JM, Quintana González JL Toribio Sánchez CM, Gómez Cisneros SC, Parra Muntaner L, García Alonso J. Utilidad del antígeno prostático específico en líquido pleural para el diagnóstico del lderrame metastático por cáncer de próstata. Actas Urol Esp 2002: 26(7)513-514. 